Aim To identify and evaluate delays in the FT process at Epsom and St Helier University Hospitals NHS Trust (ESTH). Method A previous FT audit from April 2014-March 2015 identified 61 patients who died before being discharged to their PPC. Their clinical notes were obtained and data was collected on modifiable delays.

Results Forty-seven complete sets of notes were obtained. The most significant delay was in completing and sending FT paperwork (30/47). Paperwork was not started for 3 patients and incomplete for 7 patients without explanation. Paperwork took 2-4 days to complete for 5 patients, 5-7 days for 12 patients and $>8$ days for 2 patients.

Despite healthcare professionals identifying a patient was dying or deteriorating, a delay in decision to commence FT affected 20/47 patients. The decision to FT took 1-2 days for 3 patients, 3-4 days for 8 patients, and $>8$ days for 9 patients.

Sudden unexpected death or deterioration was a factor in 8 patients. Family/patient indecision affected 8 patients. Delays within community services after funding was approved affected 5 patients. One patient had funding initially declined but agreed subsequently.

Conclusion The most significant modifiable delays were in completing FT paperwork and in decision-making concerning FT. To reduce these delays, FT paperwork on the intranet has been reorganised, new prompts in the form of a sticker have been introduced and there is continuing education of clinical teams on decision making and FT discharge.

\section{P-57 PATIENT EXPERIENCE: THE REAL STORIES}

Anne Black, Catriona Mayland, Tamsin McGlinchey, Maureen Gambles, John E Ellershaw. Marie Curie Palliative Care Institute Liverpool, University of Liverpool, Liverpool, UK

10.1136/bmjspcare-2017-00133.57

Background 'Person Centred' care has been promoted in End of Life Care since 2008 (DH 2008). National frameworks recommend that the views of service users should inform ongoing service improvement (NPELCP 2015). Therefore, a study was undertaken in one acute hospital in the North West, to explore the views and experience of recent hospital in patients, who also received direct input from the HSPCT.

Aim of this presentation Provide an overview of the project and present the main findings from this study.

Method Phenomenological approach adopted utilising:

- Narrative Interviews with 20 recently discharged palliative patients; Thematic Analysis undertaken.

- Case note review - key elements regarding recent in-patient episode.

Results Case Note Review: 'Medicalised picture' of the patient journey observed; when HSPCT involved, 'holistic' accounts were evident.

Narrative Interviews: Data collected highlighted the 'richness' of the patient experience, and the value of conducting this type of research. Five 'themes' were identified:

Institutional Cultures

- The 'simple things' matter to patients; smile, touch, compassion
Communication

- Modes of communication; impact on perceptions of care experience.

- Involvement of the HSPCT

- Initial anxiety about referral to HSPCT; fearing death was imminent.

- Therapeutic impact; focus on holistic well-being and the person as an individual.

- Loss of Control and Loss of Self

- Coming to terms with a life limiting illness; impact on sense of identity.

- Hospital provided a 'secure' and 'supportive' environment for some; once discharged some patients felt 'alone'.

- Burden of Symptoms and Treatment

- In-patient stay for some was characterised by their experience of pain.

- Challenges for patients; choices and decisions regarding treatment options.

Conclusion Findings increase knowledge of care delivery from the perspective of palliative care in-patients, and has provided information directly to the acute trust to support local change and improvements to patient care experience.

\section{P-58 THE PALLIATIVE ACADEMIC CLINICAL TRAINEES SOCIETY: DESCRIPTION AND CAREER OUTCOMES OF A NOVEL PEER SUPPORT PROGRAMME FOR ACADEMIC TRAINEES IN PALLIATIVE MEDICINE}

${ }^{1}$ Simon Noah Etkind, ${ }^{2}$ Chloe Chin, ${ }^{1}$ Natasha Lovell, 'Dominique Wakefield, ${ }^{3}$ Amara Nwosu, ${ }^{4}$ Karen Neoh, ${ }^{5}$ Guy Schofield, ${ }^{1}$ Katherine Sleeman. ${ }^{1}$ Cicely Saunders Institute, UK; ${ }^{2}$ Addenbrookes Hospital, UK; ${ }^{3}$ Marie Curie Palliative Institute Liverpool, UK; ${ }^{4}$ Leeds Teaching Hospitals NHS Trust, UK; ${ }^{5}$ Imperial College London, UK

\subsection{6/bmjspcare-2017-00133.58}

Background The UK Integrated Academic Training (IAT) pathway exists to promote the development of clinical researchers. The National Institute of Health Research (NIHR), alongside other funders, supports pre-doctoral Academic Clinical Fellow (ACF) and post-doctoral Clinical Lecturer posts for trainees across all specialties. In palliative medicine, low numbers of IATs (14 palliative medicine ACFs 2009 - 2014 compared to 114 oncology) and geographical spread mean that trainees can feel isolated. The Palliative Academic-Clinical Trainees Society (PACTs) was formed in 2012 to provide peer support and networking opportunities for this group. All palliative medicine IATs are invited to join PACTs. Annual meetings focus on peer-support/mentoring, and addressing clinical and academic training needs.

Aim To describe PACTs membership and outcomes of training for members.

Methods Evaluation of IAT career outcomes, based on an online database of PACTs membership, and comparison with national data.

Results Since inception, 23 IATs, including 17 ACFs and 6 CLs have been PACTs members. 6/9 (67\%) completed ACFs have progressed to $\mathrm{PhD}$ posts, compared to $60 \%$ of ACFs across all specialties up to 2014. 2/9 ACFs returned to clinical training and $1 / 9$ has a clinical consultant post. 1/3 completed CLs has gained an NIHR Clinician Scientist Fellowship, one 
has an honorary senior lectureship, and one has a clinical consultant post. In comparison, up to April 2014, 76\% of CLs in all specialties continued in an academic post. PACTs members have also published over 100 research papers since 2009 .

Discussion A national peer support network for IATs in palliative medicine is particularly important because the small number of posts limits local support. PACTs works well, and is considered helpful by members. Career progression for PACTs members compares favourably to national averages for IATs. This peer support model could be used elsewhere to support clinical academic training in palliative medicine.

\section{P-59 FACILITATING COLLABORATIVE WORKING WITH A PATIENT CENTRED STUDY DAY}

${ }^{1}$ Kate Shorthose, ${ }^{1}$ Anitia Brigham, ${ }^{2}$ Dawn Giblett, ${ }^{3}$ Helen Davies. 'St Margerets Hospice, Taunton, Somerset; ${ }^{2}$ Musgrove Park Hospital, Taunton, Somerset; ${ }^{3}$ Yeovil District General Hospital, Yeovil, Somerset

\subsection{6/bmjspcare-2017-00133.59}

Background A new heart failure service had recently been developed with input from the local palliative care team. Feedback from an initial joint study day was positive but felt to be too power point heavy.

A further study day was planned, with a more interactive approach. It was attended by GPs, specialist nurses and staff nurses from hospital and community settings.

Methods A programme was developed with sessions focussed around a patient's journey from diagnosis until last days of life. The sessions were led by heart failure and palliative care professionals and included a patient story. Participants were seated around tables with a facilitator on each, for group discussions about treatment decisions and specific challenges arising from the different stages of the disease.

Evaluation forms included before and after self-assessments of knowledge and confidence by the participants for each session and also asked candidates how it would change their practice.

Results $96 \%$ of participants providing feedback (25 of 26) reported an increase in confidence and better understanding of the topics covered, with improved scores before and after the teaching. The majority gave examples of changes to practice, especially around advanced care planning. Feedback was extremely positive in favour of the holistic, multidisciplinary approach and central role of the patient's story.

Conclusions The model promoted collaborative working between the palliative and heart failure teams with a better understanding of each other's role and appreciation that an integrated approach can potentially improve patient care.

Using the story of an individual patient gave more relevance and a personal aspect to the teaching sessions which was appreciated by the attendees.

There is potential for this model to be used for other noncancer patient groups such as long term neurological conditions and chronic respiratory disease to strengthen local collaborative working between palliative care and these specialities also.

\section{P-60 A PILOT SURVEY OF EDUCATIONAL AND DEVELOPMENT OPPORTUNITIES AND SUPPORT AVAILABLE TO SSAS DOCTORS WORKING IN PALLIATIVE MEDICINE IN EAST AND WEST SUSSEX}

Sara McLintock, Jane Lewington. Martlets Hospice (Sussex Community NHS Foundation Trust), Hove, UK

\subsection{6/bmjspcare-2017-00133.60}

Introduction Specialty Doctors, Staff Grade and Associate Specialist (SSAS) Doctors comprise a higher workforce percentage in palliative medicine (PM) than in other secondary care specialties (19-20\% vs 9\%). ${ }^{1,2,3}$ These grades are usually considered "non-training" but employers are expected to provide educational development support. ${ }^{4}$ The APM annual census examines workforce and workload issues ${ }^{1}$ but this survey aimed to discover what educational support is available. SSAS doctors working in East and West Sussex (EWS) were surveyed about educational development opportunities, support to access these opportunities, appraisal and future career plans.

Methods Eighteen SSAS doctors in PM in EWS were invited to complete a short online survey asking questions about educational and development opportunities.

Results Eleven people (61\%) responded to the survey, although one did not complete several questions. The main results are shown in Table 1 with figures for the rest of England shown where available.

\begin{tabular}{lll} 
Abstract P-60 Table 1 & & \\
\hline & EWS n (\%) & England (\%) \\
\hline Female & $11 / 11(100)$ & 81 \\
Less-than-full-time & $11 / 11(100)$ & 77 \\
Named supervisor & $7 / 10(70)$ & \\
Annual appraisal & $10 / 10(100)$ & 90 \\
Allocated SPA time & $9 / 10(90)$ & \\
Protected SPA time & $2 / 9(22)$ & \\
Study Leave in last 12 months & $11 / 11(100)$ & \\
Study Leave funding granted & $9 / 10(90)$ & \\
Course attendance encouraged & $9 / 10(90)$ & \\
Hospice Journal Club & $5 / 10(50)$ & \\
Access to regular teaching & $3 / 10(30)$ & \\
Would like more educational opportunities & $8 / 10(80)$ & \\
Audit/Quality Improvement project in last 2 years & $9 / 10(90)$ & \\
Plan to sit/sat exams in last 2 years & $2 / 10(20)$ & \\
Regularly teach others & $7 / 9(78)$ & \\
Attended teaching skills course & $4 / 10(40)$ & \\
Poor/no access to online journals & $3 / 10(30)$ & \\
Plan for CESR/Article 14 & $2 / 10(20)$ & \\
\hline
\end{tabular}

Discussion This pilot survey (with 61\% response rate) shows SSAS doctors in EWS are generally well supported with $90 \%$ allocated SPA time and study leave with expenses. They are educationally active doing Audit/Quality Improvement projects and teaching others. At least $2 / 10$ plan to go the CESR/Article 14 route. Areas for improvement include more/better online resource access, and locally provided teaching. Of concern, 